\title{
Optical solitons in presence of higher-order effects
}

\author{
T. Bui Dinh, ${ }^{* 1}$ V. Cao Long, ${ }^{1}$ B. Nguyen Huy, ${ }^{2}$ S. Vu Ngoc ${ }^{2}$ \\ ${ }^{I}$ Institute of Physics, University of Zielona Góra \\ ${ }^{2}$ Vinh University, Nghe An, Vietnam
}

Received April 01, 2010; revised June 18, 2010; accepted June 18, 2010; published June 30, 2010

\begin{abstract}
In this paper, we seek special soliton solutions of so-called Higher-Order Nonlinear Schroedinger Equation, which describes the propagation of light waves in nonlinear optical media. Using a developed Jacobi elliptic function expansion method described in [8], we show that in the case of four-order dispersion these traveling wave solutions do not exist. As a byproduct, we regain the results obtained by other authors [8, 9] for the case when only the dispersion to the third order is taken into account.
\end{abstract}

\section{Introduction}

It is well known that a great deal of physical processes involved in a given nonlinear problem may be understood in terms of formation of spatial, temporal or spatiotemporal localized structures or solitons. Being a true hightechnology application of these mathematical objects, optical solitons have been the objects of intensive theoretical and experimental studies in the past several decades due to their potential applications in long distance communication and all-optical ultrafast switching devices. Generally speaking, they are special solutions of the socalled Higher-Order Nonlinear Schroedinger Equation (HNLS), which describes the propagation of light waves in nonlinear optical media. As a result, it gives us the inputand-output causal relation for light waves in optical fibers. In literature, there is no systematic way of deriving the propagation equation for light waves in nonlinear media for arbitrary orders of dispersion, diffraction and nonlinearities in an arbitrary medium. In [1] we presented a method of derivation for this propagation equation in a nonlinear differential form. As a special case, we introduced this equation systematically in [2].

The study of the above-mentioned localized waves (solitons) is a difficult task, as nonlinear partial differential equations (PDEs) of a given system are usually not integrable. By investigating the integrability of a nonlinear PDE, one gains crucial insight into the structure of the equation and nature of its solutions. With the exception of some analytical solutions obtained by well-known methods (inverse scattering method, Hirota's method [5], Jacobi elliptic function expansion method [8]), solitary wave solutions have to be determined numerically [6]. One of the most effective methods is the Fourier Split-Step method which is used in studying the dynamics of short-pulse splitting in dispersive nonlinear media [3, 4]. In some cases, we should use analytical and numerical methods simultaneously [7].
In this paper, we seek special soliton solutions of HNLS, namely exact traveling wave solutions ([5], Chapter 7). As it has been emphasized in [8], these solutions are some of the most fundamental objects of study in mathematical physics. Using a developed Jacobi elliptic function expansion method described in [8], we show that in the case of four-order dispersion these traveling wave solutions do not exist. Hence, for ultrashort pulses, it is not necessary to include orders of dispersion which are higher than three to obtain solutions of this type. As a byproduct, we regain the results obtained by other authors [8,9] for the case when only the dispersion to the third order is taken into account.

Our paper is organized as follows. In Section 2, following [8] there is a short description of the developed Jacobi elliptic function expansion method. In Section 3, by using this method for the HNLS with the four-order dispersion, we show that traveling wave solutions do not exist in this case. We also regain dark and bright solitons obtained by other authors $[8,9]$. The last section contains our conclusions.

\section{Developed Jacobi elliptic function expansion}

We consider a nonlinear partial differential equation in a general form

$$
N\left(F,|F|, \frac{\partial F}{\partial t}, \frac{\partial F}{\partial x}, \frac{\partial^{2} F}{\partial t^{2}}, \frac{\partial^{2} F}{\partial x^{2}}, \frac{\partial^{2} F}{\partial x \partial t} \ldots\right)=0 .
$$

We seek the traveling wave solutions of the form

$$
F=u(\xi) e^{i(k x-\omega t)}, \xi=c x-\lambda t+x_{0},
$$

where $\mathrm{u}(\xi)$ is a real function, $\lambda$ is a constant parameter and $k$ and $\omega$ denote the wave number and frequency, respectively. Substituting (2.2) into (2.1) we obtain an ordinary differential equation

$$
N\left(u, \frac{d u}{d \xi}, \frac{d^{2} u}{d \xi^{2}}, \frac{d^{3} u}{d \xi^{3}}, \cdots\right)=0 .
$$

We take the ansatz of the solution in the form of a finite series of Jacobi elliptic functions $c n(\xi, m)($ or $\operatorname{sn}(\xi, m))$, i.e.

$$
u(\xi)=\sum_{j=0}^{n} a_{j} c n^{j}(\xi) .
$$

in which $a_{j}$ are constants which will be determined later and the highest degree of the function $u$ is 


$$
O(u(\xi))=n
$$

It follows from the properties of Jacobi elliptic functions that the highest degree of derivatives is taken as

$$
O\left(d^{p} u(\xi) / d \xi^{p}\right)=n+p .
$$

$n$ in (2.4) is selected in such a way that the highest degree of derivatives is equal to the degree of the nonlinear term. Substituting (2.4) into (2.3) and equating the coefficients of all power of $\operatorname{cn}(\xi) \operatorname{sn}(\xi), \operatorname{dn}(\xi)$ to zero leads to a set of algebraic equations for $\mathrm{a}_{\mathrm{j}}$. By solving these equations, we obtain the final result for $u$ in the form (2.4).

\section{The higher-order propagation equation}

As it has been emphasized in Sec. 1, a systematic derivation for light propagation equation in a nonlinear medium is given in $[1,2]$. In the case of ultrashort light pulses (femtosecond pulses which have much potential for future technology), in comparison with the nonlinear Schroedinger equation (NLS), higher-order terms should be taken into account. For this reason, we consider the modified NLS equation in the form

$$
\begin{aligned}
\frac{\partial E}{\partial z}= & i\left(\alpha_{1} \frac{\partial^{2} E}{\partial t^{2}}+\alpha_{2} \frac{\partial^{4} E}{\partial t^{4}}+\alpha_{3}|E|^{2} E\right)+\alpha_{4} \frac{\partial^{3} E}{\partial t^{3}}+ \\
& +\alpha_{5} \frac{\partial\left(|E|^{2} E\right)}{\partial t}+\alpha_{6} E \frac{\partial|E|^{2}}{\partial t}
\end{aligned},
$$

where the real parameters $\alpha_{i}(i=1, \ldots, 6)$ have the following physical interpretations: $\alpha_{1}$ corresponds to the group velocity dispersion (GVD), $\alpha_{2}$ to the four-order dispersion (FOD), $\alpha_{3}$ to self-phase modulation (SPM), $\alpha_{4}$ to thirdorder dispersion (TOD), $\alpha_{5}$ to self-steepening (SS) and $\alpha_{6}$ to the self frequency shift (SFS) arising from stimulated Raman scattering (SRS). In order to find traveling wave solutions of Eq.(3.1), we use the developed Jacobi elliptic function expansion method described above. Firstly, we write the electric field in the form

$$
E(z, t)=u(\xi) \exp [i .(k z-\omega . t)], \quad \xi=c t-\lambda z+z_{0} .
$$

Substituting (3.2) into (3.1) we obtain

$$
\begin{aligned}
& -\lambda u^{\prime}+i k u=i \alpha_{2}\left[c^{4} u^{\prime \prime \prime}+\omega^{4} u-6 c^{2} \omega^{2} u^{\prime \prime}+4 i\left(c \omega^{3} u^{\prime}-c^{3} \omega u^{\prime \prime \prime}\right)\right]+ \\
& +i \alpha_{1}\left(c^{2} u^{\prime \prime}-2 i c \omega u^{\prime}-\omega^{2} u\right) i \alpha_{3} u^{3}++\alpha_{5}\left(3 c u^{2} u^{\prime}-i \omega u^{3}\right)+ \\
& +\alpha_{4}\left[c^{3} u^{\prime \prime \prime}-3 c \omega^{2} u^{\prime}+i\left(\omega^{3} u-3 c^{2} \omega u^{\prime \prime}\right)\right]+2 \alpha_{6} c u^{2} u^{\prime} .
\end{aligned}
$$

Separating the real and imaginary parts of this equation leads to the following system of equations

$$
\begin{aligned}
& c^{3}\left(\alpha_{4}+4 \alpha_{2} \omega\right) u^{\prime \prime \prime}+\left(\lambda-3 \alpha_{4} c \omega^{2}+2 \alpha_{1} c \omega-4 \alpha_{2} c \omega^{3}\right) u^{\prime}+ \\
& +\left(3 \alpha_{5}+2 \alpha_{6}\right) c u^{2} u '=0, \\
& c^{4} \alpha_{2} u^{\prime \prime \prime}+\left(c^{2} \alpha_{1}-6 \alpha_{2} c^{2} \omega^{2}-3 c^{2} \omega \alpha_{4}\right) u^{\prime \prime}+ \\
& +\left(-k-\alpha_{1} \omega^{2}+\alpha_{2} \omega^{4}+\alpha_{4} \omega^{3}\right) u+\left(\alpha_{3}-\alpha_{5} \omega\right) u^{3}=0
\end{aligned}
$$

Now using developed Jacobi elliptic function expansion method described in Sec. 2 we calculate the highest degree of derivative $O\left(d^{4} u(\xi) / d \xi^{4}\right)=n+4$ and the degree of the nonlinear term $O\left(u^{3}(\xi)\right)=3 n$. Equating these numbers leads to $n=2$. It follows that the function $u(\xi)$ can be obtained from the form

$$
u(\xi)=a_{0}+a_{1} c n(\xi)+a_{2} c n^{2}(\xi) .
$$

For the sake of simplicity, we suppose that $a_{0}=a_{1}=0$, then $\mathrm{u}(\xi)=\mathrm{a}_{2} \mathrm{cn}^{2}(\xi)$. Substituting this expression into Eq. (3.4b) gives

$$
A_{6} c n^{6}(\xi)+A_{4} c n^{4}(\xi)+A_{2} c n^{2}(\xi)+A_{0}=0,
$$

where coefficients $A_{i}$ contain different parameters involved in the problem. Equating the coefficient of the first term in (3.6) to zero leads to $A_{6}=48 \alpha_{2} c^{4} a_{2} m^{4}+24 \alpha_{2} c^{4} a_{2} m^{2}=0$. Because $a_{2}, m, c$ should be different from zero, we have $\alpha_{2}=0$. This means that if the term FOD is taken into account, the traveling wave solutions do not exist. We conclude that for the existence of solutions in this type, the orders of dispersion higher than three should not be taken into account. Then we can rewrite (3.4a) and (3.4b) in the form (with $\alpha_{2}=0$ )

$$
c^{3} \alpha_{4} u^{\prime \prime \prime}+\left(\lambda-3 \alpha_{4} c \omega^{2}+2 \alpha_{1} c \omega\right) u^{\prime}+\left(3 \alpha_{5}+2 \alpha_{6}\right) c u^{2} u^{\prime}=0
$$

$\left(c^{2} \alpha_{1}-3 c^{2} \omega \alpha_{4}\right) u^{\prime \prime}+\left(-k-\alpha_{1} \omega^{2}+\alpha_{4} \omega^{3}\right) u+\left(\alpha_{3}-\alpha_{5} \omega\right) u^{3}=0$

Differentiating two sides of Eq. (3.7b) with respect to the $\xi$ gives us

$$
u^{\prime \prime \prime}+\frac{\left(-k-\alpha_{1} \omega^{2}+\alpha_{4} \omega^{3}\right)}{\left(c^{2} \alpha_{1}-3 c^{2} \omega \alpha_{4}\right)} u^{\prime}+3 \frac{\left(\alpha_{3}-\alpha_{5} \omega\right)}{\left(c^{2} \alpha_{1}-3 c^{2} \omega \alpha_{4}\right)} u^{2} u^{\prime}=0 .
$$

Comparing (3.8) with (3.7a) leads to formulas for $\omega$ and $k$ :

$$
\begin{array}{r}
\omega=\left[\alpha_{1}\left(3 \alpha_{5}+2 \alpha_{6}\right)-3 \alpha_{3} \alpha_{4}\right]\left[6 \alpha_{4}\left(\alpha_{5}+\alpha_{6}\right)\right]^{-1}, \\
k=-\frac{1}{c \alpha_{4}}\left(\lambda-3 \alpha_{4} c \omega^{2}+\alpha_{1} c \omega\right)-\alpha_{1} \omega^{2}+\omega^{3} \alpha_{4} .
\end{array}
$$

Then Eqs. (3.7a), (3.7b) reduce to

$$
u^{\prime \prime}+A u+B u^{3}=0 \text {, }
$$

where

$$
A=\frac{2 \alpha_{1} \omega+\lambda-3 \alpha_{4} \omega^{2}}{c^{2} \alpha_{4}} ; \quad B=\frac{3 \alpha_{5}+2 \alpha_{6}}{3 c^{2} \alpha_{4}} .
$$

Now, we use the formalism described in Sec.2 for the Eq.(3.11). Firstly, we calculate $O\left(d^{2} u / d \xi^{2}\right)=n+2$ and $O\left(u^{3}(\xi)\right)=3 n$. Then $\mathrm{n}=1$ and we can write $\mathrm{u}(\xi)$ in the following form:

$$
u(\xi)=a_{0}+a_{1} c n(\xi) \text {. }
$$

Substituting (3.13) into (3.11) and equating the coefficients of all powers of $\mathrm{cn}(\xi)$ to zero yields the values of unknown parameters $a_{0}, a_{1}, c, \lambda$. We have performed this step by MAPLE and obtained:

$a_{0}=0, a_{1}=\sqrt{\frac{6 \alpha_{4}}{2 \alpha_{6}+3 \alpha_{5}}} m c, \lambda=-2 m^{2} c^{2} \alpha_{4}-2 \alpha_{1} \omega+c^{2} \alpha_{4}+3 \alpha_{4} \omega^{2}$,

while $c$ is an arbitrary constant and $m$ is the modulus number of the Jacobi elliptic functions. Then the traveling wave solutions of the propagation equation (3.1) have the following form 


$$
\begin{aligned}
& E(z, t)=\sqrt{\frac{6 \alpha_{4}}{2 \alpha_{6}+3 \alpha_{5}}} m c \cdot \exp [i .(k z-\omega t)] . \\
& . c n\left[c t-\left(-2 m^{2} c^{2} \alpha_{4}-2 \alpha_{1} \omega+c^{2} \alpha_{4}+3 \alpha_{4} \omega^{2}\right) z+z_{0}\right]
\end{aligned}
$$

where the expressions for $\mathrm{k}, \omega$ are given by (3.9) and (3.10). When $m$ tends to 1 , we obtain a bright soliton solution

$$
\begin{aligned}
& E(z, t)=\sqrt{\frac{6 \alpha_{4}}{2 \alpha_{6}+3 \alpha_{5}}} c \cdot \exp [i .(k z-\omega t)] . \\
& . \sec h\left[c t-\left(-c^{2} \alpha_{4}-2 \alpha_{1} \omega+3 \alpha_{4} \omega^{2}\right) z+z_{0}\right] .
\end{aligned}
$$

Now instead of (3.13) we use the ansatz

$$
u(\xi)=a_{0}+a_{1} \operatorname{sn}(\xi) \text {. }
$$

By substituting (3.17) into (3.11) we obtain $a_{0}=0, a_{1}=\sqrt{\frac{-6 \alpha_{4}}{2 \alpha_{6}+3 \alpha_{5}}} m c, \lambda=c^{2} \alpha_{4}-2 \alpha_{1} \omega+m^{2} c^{2} \alpha_{4}+3 \alpha_{4} \omega^{2}$,

while $c$ is also an arbitrary constant. Then the solution of Eq.(3.1) has the following form:

$$
\begin{aligned}
E(z, t) & =\sqrt{\frac{-6 \alpha_{4}}{2 \alpha_{6}+3 \alpha_{5}}} m c \cdot \exp [i \cdot(k z-\omega t)] . \\
& . s n\left[c t-\left(c^{2} \alpha_{4}-2 \alpha_{1} \omega+m^{2} c^{2} \alpha_{4}+3 \alpha_{4} \omega^{2}\right) z+z_{0}\right]
\end{aligned}
$$

where the expressions for $\mathrm{k}, \omega$ are given by (3.9) and (3.10). When the modulus number $m$ tends to 1 we have a dark soliton solution in the following form:

$$
\begin{aligned}
E(z, t)= & \sqrt{\frac{-6 \alpha_{4}}{2 \alpha_{6}+3 \alpha_{5}}} c \cdot \tanh \left[c t-\left(2 c^{2} \alpha_{4}-2 \alpha_{1} \omega+3 \alpha_{4} \omega^{2}\right) z+z_{0}\right] \\
& . \exp [i .(k z-\omega t)]
\end{aligned}
$$

Our expressions (3.16) and (3.20) are just the results previously obtained by several authors (e.g. the formulas (56), (58) in [8] and (9), (12) in [9]). We demonstrate these for some values of parameters involved in Figures 1-4.

\section{Conclusions}

In our paper we used a developed Jacoby elliptic function expansion method to find traveling wave solutions for HNLS which describes light propagation in fibers. We showed that we cannot have solutions of this type when we include higher orders of dispersion. As a byproduct, we obtained in a simple way bright and dark soliton solutions discovered before by other authors.

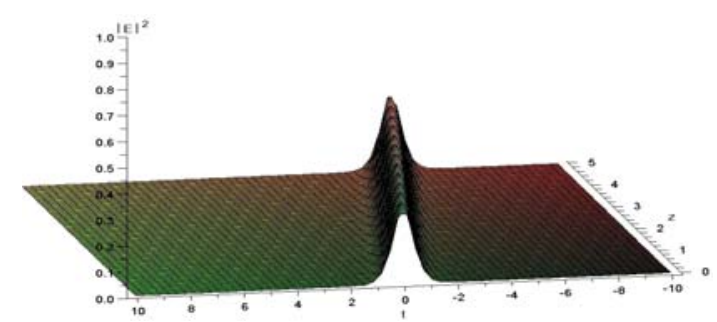

Fig. 1: Bright soliton (3.16) with c $=2, \alpha_{1}=-0.5, \alpha_{3}=1, \alpha_{4}=1 / 24, \alpha_{5}=0.8$, $\alpha_{6}=0.5$ and $\mathrm{z}_{0}=0$.

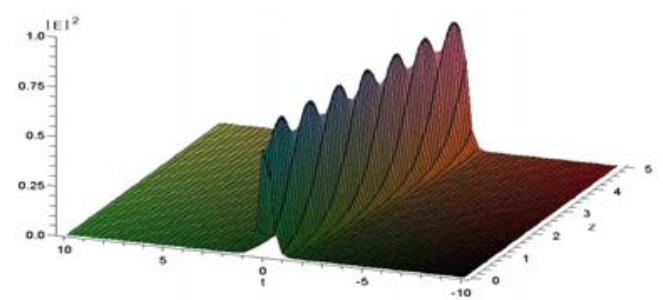

Fig.2. Bright soliton (3.16) with c $=3, \alpha_{1}=-0.5, \alpha_{3}=1, \alpha_{4}=1 / 24, \alpha_{5}=0.8$, $\alpha_{6}=0.5$ and $\mathrm{z}_{0}=0$.

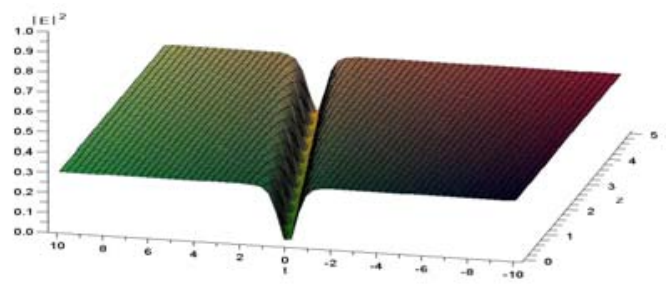

Fig.3. Dark soliton (3.19) with c $=2, \alpha_{1}=-0.5, \alpha_{3}=1, \alpha_{4}=-1 / 24, \alpha_{5}=0.8$, $\alpha_{6}=0.5$ and $\mathrm{z}_{0}=0$.

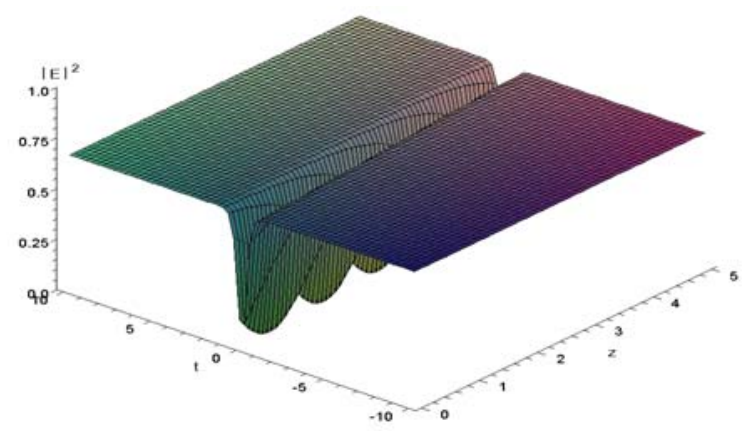

Fig.4. Dark soliton (3.19) with c $=3, \alpha_{1}=0.5, \alpha_{3}=1, \alpha_{4}=-1 / 24, \alpha_{5}=0.8$, $\alpha_{6}=0.5$ and $\mathrm{z}_{0}=0$.

\section{References}

[1] C. Long Van, M. Trippenbach, New Optical Solitary waves in the higher order nonlinear Schroedinger Equation, Scientific Bulletin 126, Technical University of Zielona Gora, Poland 2001, p.19 (Cao Long Van ed.).

[2] V. Cao Long, H. Nguyen Viet, M. Trippenbach, K. Dinh Xuan, Computational Methods in Science and Technology 14(1), 5 (2008).

[3] V. Cao Long, H. Nguyen Viet, M. Trippenbach, K. Dinh Xuan, Computational Methods in Science and Technology 14(1), 13 (2008).

[4] V. Cao Long, H. Nguyen Viet, M. Trippenbach, K. Dinh Xuan, Computational Methods in Science and Technology 14(1), 21 (2008).

[5] C. Long Van, P. P. Goldstein, A concise Course in nonlinear Partial Differential Equations (University of Zielona Gora 2008).

[6] A. V. Buryak and Yu. S. Kivshar, Phys. Lett. A 197, 407 (1995).

[7] M. Trippenbach, M. Matuszeski, E. Infreld, Cao Long Van, R. S. Tasgal, J. B. Band, Optics Comm. 229, 391 (2004).

[8] S. A. El-Wakil, M.A. Abdou, A. Elhanbaly, Phys. Lett. A 353, 40 (2006).

[9] Q. Yang, C.Q. Dai, J. F. Zhang, International Journal of Theoretical Physics 44, 1117 (2005). 